\title{
Therapeutic Effect of Dunaliella salina Microalgae on Thioacetamide- (TAA-) Induced Hepatic Liver Fibrosis in Rats: Role of TGF- $\beta$ and MMP9
}

\author{
Farouk K. El-Baz, ${ }^{1}$ Abeer Salama ${ }^{(1)}{ }^{2}$ and Rania A. A. Salama ${ }^{3}$ \\ ${ }^{1}$ Plant Biochemistry Department, National Research Centre (NRC), 33 El Bohouth St. (Former El-Tahrir St.), 12622 Dokki, \\ Giza, Egypt \\ ${ }^{2}$ Pharmacology Department, National Research Centre (NRC), 33 El Bohouth St. (Former El-Tahrir St.), 12622 Dokki, \\ Giza, Egypt \\ ${ }^{3}$ Toxicology and Narcotics Department, National Research Centre (NRC), 33 El Bohouth St. (Former El-Tahrir St.), 12622 Dokki, \\ Giza, Egypt
}

Correspondence should be addressed to Abeer Salama; berrotec@yahoo.com

Received 7 July 2019; Accepted 27 August 2019; Published 24 September 2019

Academic Editor: Hongqun Liu

Copyright (c) 2019 Farouk K. El-Baz et al. This is an open access article distributed under the Creative Commons Attribution License, which permits unrestricted use, distribution, and reproduction in any medium, provided the original work is properly cited.

\begin{abstract}
Liver fibrosis represents a serious global health-care problem and is the outcome of many chronic liver diseases, cirrhosis, hepatitis, and toxin accumulation. The present study aimed to evaluate the antifibrotic curative effect of Dunaliella salina (D. salina) on thioacetamide- (TAA-) induced liver fibrosis in rats. Liver fibrosis was induced by TAA (200mg/kg; i.p.) twice per week for 6 weeks. D. salina was given orally $(100$ and $200 \mathrm{mg} / \mathrm{kg})$ and silymarin was given orally $(100 \mathrm{mg} / \mathrm{kg})$ daily, for 4 weeks after TAA. Serum transaminase activities, liver inflammatory cytokines, fibrotic biomarkers, and liver histopathology were assessed. TAA significantly $(p<0.05)$ elevated serum activities of aspartate aminotransferase (AST) and alanine aminotransferase (ALT) and serum levels of total bilirubin, tumor necrosis factor-alpha (TNF- $\alpha$ ), interleukin-6 (IL-6), and transforming growth factorbeta (TGF- $\beta$ ) with a reduction in albumin. In addition, TAA increased hepatic contents of collagen I, $a$-smooth muscle actin ( $\alpha$-SMA), and tissue inhibitors of metalloproteinase (TIMP-1), reduced matrix metalloproteinase 9 (MMP9), and finally produced marked degeneration and fibrosis of hepatocytes. Treatment with D. salina or silymarin improved the histological feature of hepatocytes and ameliorated the deleterious effects of TAA in a dose-dependent manner. Based on these results, it could be concluded that the use of $D$. salina could be assigned for liver fibrosis treatment via its anti-inflammatory and antifibrotic properties.
\end{abstract}

\section{Introduction}

Liver fibrosis is a health problem that leads to morbidity and mortality and is usually insidious [1]. Liver fibrosis results from chronic damage to the liver in conjunction with accumulation of extracellular matrix (ECM) proteins, which is a characteristic of most types of chronic liver diseases. The accumulation of ECM proteins distorts the hepatic architecture by forming a fibrous scar, and the subsequent development of nodules of regenerating hepatocytes defines cirrhosis as provoking hepatic failure or hepatocellular carcinoma [2]. Liver fibrosis is thought to be a process of hepatic parenchyma and collagen collapse, and it may happen as a result of some etiological factors such as alcohol consumption, obesity, metabolic disorders, cholestasis, steatosis, viral infection, and toxin accumulation [3].

ECM controls cellular functions and its dysregulation linked with liver disease development [4]. Matrix metalloproteinases (MMPs), endopeptidases, destroy ECM components and degrade proteins [5]. They also have an important role on non-ECM substrates, like cytokines, regulating immunity and inflammation [6]. However, 
MMPs proteolytic activities are suppressed by tissue inhibitors of metalloproteinases (TIMPs) in tissues [7].

Single administration of thioacetamide (TAA), a thiosulfur compound, induced acute hepatic toxicity while hepatic cirrhosis or liver tumors developed by chronic exposure to it [8]. Lacking of safe drugs for the treatment of liver diseases is of considerable interest in finding natural products used in medicinal materials or foods as reported by the World Health Organization [9]. Medicinal plant extracts had gained increasing interest as nondrug remedy for many diseases. This may be due to their active constituents that modulate oxidative stress and inflammatory and cell damage [10]. As fibrogenesis increases, the key challenge will be translating into the development of antifibrotic drugs for patients with chronic liver disease [11].

In recent years, natural carotenoids have health benefits that attract attention, and these benefits were gained from carotenes and xanthophylls that have the ability to scavenge oxygen radicals [12]. Microalgae are the richest sources of natural carotenoids, especially strains of Chlorophyta such as Dunaliella salina (D. salina) which is a biflagellate unicellular green alga [13-15]. The algal cells produced three valuable products: $\beta$-carotene, glycerol, and proteins. $\beta$-carotene, an antioxidant, is used as a provitamin A food supplement and coloring agent and also used in cosmetics and a health product [16].

The aim of this study was to investigate the therapeutic effect of $D$. salina microalgae on TAA-induced hepatic liver fibrosis in rats. In addition, the role of TGF- $\beta$ and MMP9 was also investigated in this study.

\section{Materials and Methods}

2.1. Chemicals. Thioacetamide (TAA) was purchased from Sigma-Aldrich, USA. Silymarin was obtained from MEPACO, Egypt; all other chemicals, used throughout the experiment, were of the highest analytical grade available.

2.2. Animals. Adult male albino Wistar rats weighing 150-200 gm were obtained from the animal house at the National Research Centre (Giza, Egypt) and fed a standard laboratory diet and tap water ad libitum. Experimental animals were housed in an air-conditioned room at $22-25^{\circ} \mathrm{C}$ with a $12 \mathrm{~h}$ light/dark cycle. All animals received human care, and the study protocols were carried out according to the ethical guidelines for care and use of experimental animals approved by the Ethical Committee of the National Research Centre.

2.3. Cultivation of D. salina in the Vertical Photobioreactor. Algal species D. salina isolated from the Nile River water and salt pond in Al-Fayoum are grown by using BG11 media for algal isolation and purification (Stanier et al., 1971). After growing D. salina for 10 days under lab conditions, they were then transferred to a vertical photobioreactor with a capacity of $4000 \mathrm{~L}$. Reservoir (1000 L) tank associated pipe work proprietary in line pigging systems and removed all biofilms. In addition, a $10 \mathrm{~L}$ basket centrifuge for harvesting was connected to the system. The Alga Connect Data Acquisition System used for online measurements.

Tap water was used for the cultivation of algae in the PBR. Water was sterilized using hypochlorite after which sodium thiosulpate was added. Chlorine test was performed to insure no residual chlorine was present. Nutrient solution of BG 11 was used for growing D. salina. One millilitre of micronutrient solution was added to the culture medium. To insure the purity of the culture, samples were taken regularly and examined microscopically. The culture was left to grow until the biomass reached the maximum (2-2.5 gm/L). Algal biomass was harvested using a basket centrifuge at $2000 \mathrm{rpm}$, sun-dried where the temperature reached approximately $45^{\circ} \mathrm{C}$, and then ground into homogeneous fine powder.

2.4. Experimental Design. After an acclimatization period of one week, male albino Wistar rats were randomly assigned to 5 groups of 8 rats per group as follows. Group 1: received water containing $0.1 \%$ Tween 80 orally for 6 weeks and served as normal control. Group 2: received TAA (200 mg/kg, i.p.) twice per week for 6 weeks and served as TAA control according to Bai et al. [17] and Afifi et al. [18]. Group 3: received silymarin $(100 \mathrm{mg} / \mathrm{kg}$, orally) [19] daily for 4 weeks after TAA. Groups 4 and 5: received D. salina powder (100 and $200 \mathrm{mg} / \mathrm{kg}$, orally) $[20,21]$ daily for 4 weeks after TAA.

2.5. Collection of Blood Samples. At the end of the experimental period, blood samples were withdrawn from the retroorbital vein of each animal, under mild anesthesia with diethyl ether. Blood was allowed to coagulate and then centrifuged at $3000 \mathrm{rpm}$ for $15 \mathrm{~min}$. The obtained serum was used to estimate the activities of aspartate transaminase (AST) and alanine transaminase (ALT) and levels of total bilirubin and albumin using the Biodiagnostic kit, Egypt. Tumor necrosis factor-alpha (TNF- $\alpha$ ), interleukin-6 (IL-6), transforming growth factor (TGF- $\beta$ ), collagen I, alpha-smooth muscle actin ( $\alpha$-SMA), metalloproteinase-9 (MMP9), and tissue inhibitors of metalloproteinase-1 (TIMP-1) were determined using ELIZA kits procured from SinoGeneClon Biotech Co., Ltd.

2.6. Histological Examination. At the end of the experiment, part of the liver was removed carefully and fixed in $10 \%$ formalin for 24 hours. The samples were washed under tap water, dehydrated in ascending grades of ethanol (50, 70, 80, 90 , and $100 \%)$, cleared in xylene, and embedded in paraffin wax (melting point $55-60^{\circ} \mathrm{C}$ ). Liver sections of $4 \mu \mathrm{m}$ thickness were prepared and stained with haematoxylin and eosin. The paraffin sections were stained in Harris's haematoxylin for 5 minutes. Sections were washed in running water for blueing and then stained in $1 \%$ watery eosin for 2 minutes, washed in water, dehydrated, cleared, and mounted using Canada balsam. Other parts of the liver were stained with Masson's trichrome.

Eight microscopic fields per section were examined using a light microscope (Olympus BX50, Japan) under 
high-power magnification $(\times 400)$. Fibrosis staging was defined as 0 , no fibrosis; 1 , perisinusoidal and/or pericentral; 2 , incomplete central/central bridging fibrosis; 3, portalportal or portal-central fibrosis; and 4, cirrhosis [22].

2.7. Statistical Analysis. All the values are presented as means \pm standard error of the means (SE). Data of this study were evaluated by one-way analysis of variance followed by Tukey's multiple comparisons test. Graphpad Prism software, version 5 (Inc., San Diego, USA) was used to carry out these statistical tests. The difference was considered significant when $p<0.05$.

\section{Results}

3.1. D. salina Ameliorates Liver Function Changes of TAAInduced Fibrosis. Injection of TAA, for 6 weeks, produced hepatic fibrosis evidenced by significant increases in serum AST, ALT activities, and bilirubin level and decreased albumin level as compared to normal control values. Treatment with a low dose of $D$. salina powder, for 4 weeks, reduced serum activities of ALT and AST only, while treatment with a high dose of $D$. salina powder decreased serum activity of AST and normalized serum ALT activity and bilirubin and albumin levels, as compared to the TAA group. Treatment with silymarin, for 4 weeks, decreased serum activity of AST and returned serum ALT activity and bilirubin and albumin levels to normal values, as compared to the TAA group (Table 1).

\subsection{D. salina Inhibits TAA-Induced Inflammatory Biomarkers} of Fibrosis. Hepatic inflammation induced, after 6 weeks, by injection of TAA was evidenced by significant increases in serum levels of TNF- $\alpha$, IL- 6 , and TGF- $\beta$ as compared to normal control values. Treatment with both doses of $D$. salina powder, for 4 weeks, reduced serum levels of TGF- $\beta$ and returned TNF- $\alpha$ and IL- 6 to their normal value, as compared to the TAA group. Treatment with silymarin, for 4 weeks, normalized IL- 6 serum levels and decreased serum levels of TNF- $\alpha$ and TGF- $\beta$, as compared to the TAA group (Table 2).

\subsection{D. salina Inhibits TAA-Induced Fibrotic Biomarkers of} Fibrosis. TAA injection provoked hepatic fibrosis, after 6 weeks, and significantly elevated hepatic contents of collagen $\mathrm{I}$ and $a$-SMA and reduced MMP9 as compared to normal control values. In addition, treatment with both doses of $D$. salina powder, for 4 weeks, returned hepatic contents of collagen I and $a$-SMA to their normal values, while treatment with a high dose of $D$. salina powder elevated the MMP-9 hepatic content and normalised TIMP hepatic content, as compared to the TAA group. Treatment with silymarin, for 4 weeks, reduced the hepatic contents of collagen I and a-SMA, elevated those of MMP-9 and normalised TIMP hepatic content, as compared to the TAA group (Figure 1).

3.4. D. salina Improves Liver Tissue against TAA-Induced Fibrosis. The scoring of liver fibrosis in the TAA group, after
6 weeks, revealed that the fibrosis model was successfully established as compared to the healthy control group. However, the scoring of liver fibrosis in $D$. salina powder groups after 4 weeks treatment indicated that $D$. salina intervention was effective to treat fibrosis as compared to the TAA group (Figure 2).

The liver of normal control rat showed the normal histological structure of hepatic lobule (stage F0) (Figure 3(a)). The liver of rat from the TAA group showed ballooning degeneration of hepatocytes (small arrow), fibrosis of hepatocytes (large arrow), and mononuclear cells infiltration (arrow head) and $\mathrm{E} \times 400$ (stage F3) (Figure 3(b)). The liver of rat from the TAA group showed portal fibrosis (small arrow) with oval cells proliferation (large arrow) (stage F3) (Figure 3(c)). The liver of rat from the silymarin-treated group showed fine strands of fibroblasts in the portal tract (arrow) (stage F1) (Figure 3(d)). The liver of rat treated with $D$. salina $(100 \mathrm{mg} / \mathrm{kg})$ showed fatty change of hepatocytes (small arrow) and portal infiltration with inflammatory cells (large arrow) (stage F1) (Figure 3(e)). The liver of rat treated D. salina $(200 \mathrm{mg} / \mathrm{kg})$ showed slight hydropic degeneration of hepatocytes (small arrow) and portal infiltration with inflammatory cells (large arrow) (stage F1) (Figure 3(f)) (H\&E ×400).

The liver of control rat showed no histochemical reaction for collagen fibers deposition in the portal triad (Masson's trichrome stain $\times 400$ ) $($ F0) (Figure $4(\mathrm{a})$ ). The liver of rat from the TAA group showed strong positive histochemical reaction for collagen fibers deposition in the portal triad (F3) (Figure 4(b)). The liver of rat from the TAA group showed strong positive histochemical reaction for collagen fibers deposition in the portal triad (F3) (Figure 4(c)). The liver of rat treated with silymarin showed weak positive histochemical reaction for collagen fibers deposition (F1) (Figure 4(d)). The liver of rat treated with D. salina $(100 \mathrm{mg} / \mathrm{kg})$ showed weak positive histochemical reaction for collagen fibers deposition (F1) (Figure 4(e)). The liver of rat treated with $D$. salina $(200 \mathrm{mg} / \mathrm{kg})$ showed no histochemical reaction for collagen fibers deposition (F0) (Figure 4(f)) (Masson's trichrome stain $\times 400$ ).

\section{Discussion}

TAA is a hepatotoxin and used to evaluate liver fibrogenesis mechanisms [23]. One of the mechanisms underlying fibrogenesis is the liberation of reactive oxygen species (ROS), provoking oxidative stress [24]. Here, in this study, TAA produced a liver damage experimental model to mimic pathophysiological symptoms of human liver diseases via the injury of liver cell membrane, elevating activities of ALT, AST, and bilirubin level associated with a reduction in albumin compared to the control. On the contrary, treatment with $D$. salina or silymarin reduced serum activities of ALT and AST preserving hepatocyte integrity. It is worthy to note that $D$. salina also decreased the total bilirubin serum level with an increase in albumin compared to the TAA control group. Moreover, the decrease in the serum level of bilirubin after treatment with $D$. salina indicates the effectiveness in normalizing the functional state of the liver. These results come in line with those of Kumar et al. who showed that 
TABLE 1: Effects of $D$. salina powder on serum hepatic function biomarkers.

\begin{tabular}{|c|c|c|c|c|c|}
\hline & $\begin{array}{l}\text { Normal } \\
\text { control }\end{array}$ & TAA & $\begin{array}{l}\text { Silymarin } \\
(100 \mathrm{mg} / \mathrm{kg})\end{array}$ & $\begin{array}{c}\text { D. salina } \\
\text { powder } \\
(100 \mathrm{mg} / \mathrm{kg})\end{array}$ & $\begin{array}{c}\text { D. salina } \\
\text { powder } \\
(200 \mathrm{mg} / \mathrm{kg})\end{array}$ \\
\hline$\overline{A L T}(\mathrm{U} / \mathrm{L})$ & $43.14 \pm 0.18$ & $68.52 \pm 0.62^{\mathrm{a}}$ & $47.95 \pm 0.05^{\mathrm{b}}$ & $53.70 \pm 0.04^{\mathrm{ab}}$ & $48.64 \pm 0.12^{\mathrm{b}}$ \\
\hline$\%$ of TAA control & & & 70 & 78 & 71 \\
\hline AST (U/L) & $56.48 \pm 0.04$ & $89.01 \pm 0.26^{\mathrm{a}}$ & $68.56 \pm 0.37^{\mathrm{ab}}$ & $72.78 \pm 0.15^{\mathrm{ab}}$ & $69.28 \pm 0.03^{\mathrm{ab}}$ \\
\hline$\%$ of TAA control & & & 77 & 82 & 78 \\
\hline Bilirubin (mg/dl) & $1.44 \pm 0.11$ & $1.89 \pm 0.01^{\mathrm{a}}$ & $1.56 \pm 0.06^{\mathrm{b}}$ & $1.62 \pm 0.06$ & $1.55 \pm 0.07^{\mathrm{b}}$ \\
\hline$\%$ of TAA control & & & 83 & 86 & 82 \\
\hline Albumin $(\mathrm{g} / \mathrm{dl})$ & $4.13 \pm 0.03$ & $3.56 \pm 0.14^{\mathrm{a}}$ & $3.90 \pm 0.05^{\mathrm{b}}$ & $3.59 \pm 0.00^{\mathrm{a}}$ & $3.91 \pm 0.01^{\mathrm{b}}$ \\
\hline$\%$ of TAA control & & & 110 & 101 & 110 \\
\hline
\end{tabular}

Data are presented as mean \pm SE for each group $(n=8)$ and $\%$ of the TAA group. Statistical analysis was carried out by one-way analysis of variance followed

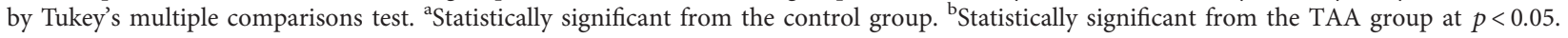

TABLE 2: Effects of D. salina powder on serum levels of inflammatory biomarkers.

\begin{tabular}{|c|c|c|c|c|c|}
\hline & $\begin{array}{c}\text { Normal } \\
\text { control }\end{array}$ & TAA & $\begin{array}{l}\text { Silymarin } \\
(100 \mathrm{mg} / \mathrm{kg})\end{array}$ & $\begin{array}{c}\text { D. salina } \\
\text { powder } \\
(100 \mathrm{mg} / \mathrm{kg})\end{array}$ & $\begin{array}{c}\text { D. salina } \\
\text { powder } \\
(200 \mathrm{mg} / \mathrm{kg})\end{array}$ \\
\hline TNF- $\alpha(\mathrm{Pg} / \mathrm{ml})$ & $600.00 \pm 8.94$ & $808.00 \pm 17.15^{\mathrm{a}}$ & $690.00 \pm 26.83^{\mathrm{ab}}$ & $655.00 \pm 11.18^{\mathrm{b}}$ & $610.00 \pm 4.47^{\mathrm{b}}$ \\
\hline TNF- $\alpha$ (\% of TAA control) & & & 85 & 81 & 75 \\
\hline IL-6 $(\mathrm{Pg} / \mathrm{ml})$ & $69.40 \pm 4.73$ & $313.80 \pm 28.34^{\mathrm{a}}$ & $101.80 \pm 0.80^{\mathrm{b}}$ & $71.20 \pm 6.02^{\mathrm{b}}$ & $69.62 \pm 4.74^{\mathrm{b}}$ \\
\hline IL-6 (\% of TAA control) & & & 32 & 23 & 22 \\
\hline TGF- $\beta(n g / L)$ & $34.33 \pm 0.70$ & $65.07 \pm 0.80^{\mathrm{a}}$ & $40.30 \pm 1.38^{\mathrm{ab}}$ & $47.47 \pm 1.27^{\mathrm{ab}}$ & $39.80 \pm 0.20^{\mathrm{ab}}$ \\
\hline TGF- $\beta$ ( $\%$ of TAA control) & & & 62 & 73 & 61 \\
\hline
\end{tabular}

Data are presented as mean \pm SE for each group $(n=8)$ and $\%$ of the TAA group. Statistical analysis was carried out by one-way analysis of variance followed

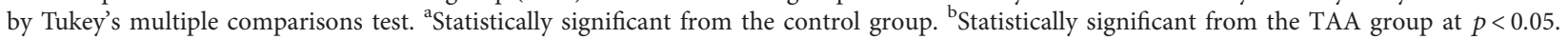

TAA injection exhibited liver dysfunction signs evidenced by serum elevation of ALT and AST activities [25]. Moreover, Galisteo et al. found that the injection of rats with TAA produces a significant decrease in plasma albumin in relation to the control normal group [26]. Moreover, Hsu et al. revealed that $D$. salina has ameliorative effects on hepatotoxicity induced by carbon tetrachloride $\left(\mathrm{CCl}_{4}\right)$, decreasing serum activities of ALT and AST [27].

Liver fibrosis is considered as a wound-healing response and characterized by progressive inflammation, stimulating ECM deposition and formation of tissue scar. In the fibrotic liver, the principal collagen-producing cells include activated hepatic stellate cells (HSCs), portal fibroblasts, and myofibroblasts [28]. Activated myofibroblasts have an important role in forming fibrotic tissue [29]. In addition, Kupffer cells, hepatocytes, sinusoidal endothelial cells, and lymphocytes may contribute to liver fibrosis [30].

One of the first manifestations of liver fibrosis is upregulation of proinflammatory cytokine release such as TNF- $\alpha$ and IL- $1 \beta$ by Kupffer cells and stimulated HSCs. TGF- $\beta$ has multiple profibrogenic effects differentiating HSCs into myofibroblasts [31]. In the present study, TAA injection, after 6 weeks, induced liver inflammation detected by a significant increase in the cytokine levels of TNF- $\alpha$, IL-6, and TGF- $\beta$ in serum compared to the normal control group. On the contrary, treatment with $D$. salina showed a significant decrease in TNF- $\alpha$, IL- 6 , and TGF- $\beta$ serum contents as compared to the TAA control group. In liver injury experimental models, TNF- $\alpha$ and IL- 6 are inflammatory mediators which affect organ function homeostasis and produce hepatotoxicity. A pervious study exhibited TAA-induced acute liver injury through farnsoid $\mathrm{X}$ receptor AMP-activated protein kinase (FXR/AMPK) signal pathway inducing oxidative stress and inflammation in rats and mice [32]. The injection of rats with TAA produced a significant elevation in the serum level of TNF- $\alpha$ and IL-6 in TAA-injected rats in comparison with those in the normal control group.

The progression of fibrogenesis leads to increased synthesis of collagen [33]. The activation of quiescent HSCs and their differentiation into myofibroblast-like cells controlled by immune cells released inflammatory cytokines and some growth factors secreting ECM contents, mainly, collagen I, III, and IV, and expressing $\alpha$-SMA. Collagen accumulation is a mark of fibrosis in the liver [34]. Our results exhibited that TAA induced a significant increase in hepatic collagen I and $\alpha$-SMA, while treatment with $D$. salina resulted in a significant decrease in collagen I and $\alpha$-SMA. In addition, during fibrogenesis, in the current work, the ECM is reworked resulting in elevations of hepatic contents of collagen I and TIMP1 coupled with a reduction in MMP-9 in the TAA group. These results explain that MMPs degrade collagen and MMPs with MMP inhibitors (TIMPs) play a key role in fibrogenesis and fibrolysis. MMP-9 is the gelatinase secreted by Kupffer cells and is implicated in the pathological process of hepatic injury [35]. Moreover, treatment with $D$. salina produced an increase in the serum concentration level of MMP-9 associated with a decrease in serum TIMP-1 compared to 


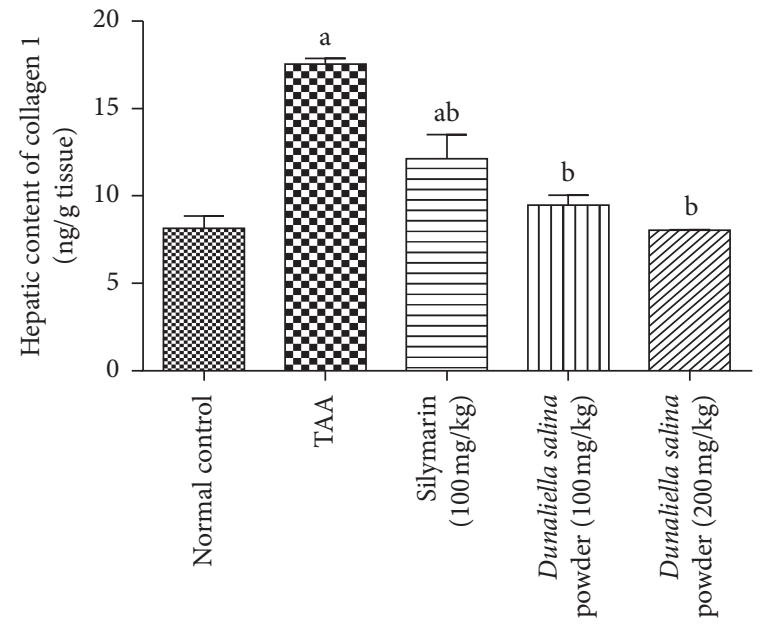

(a)

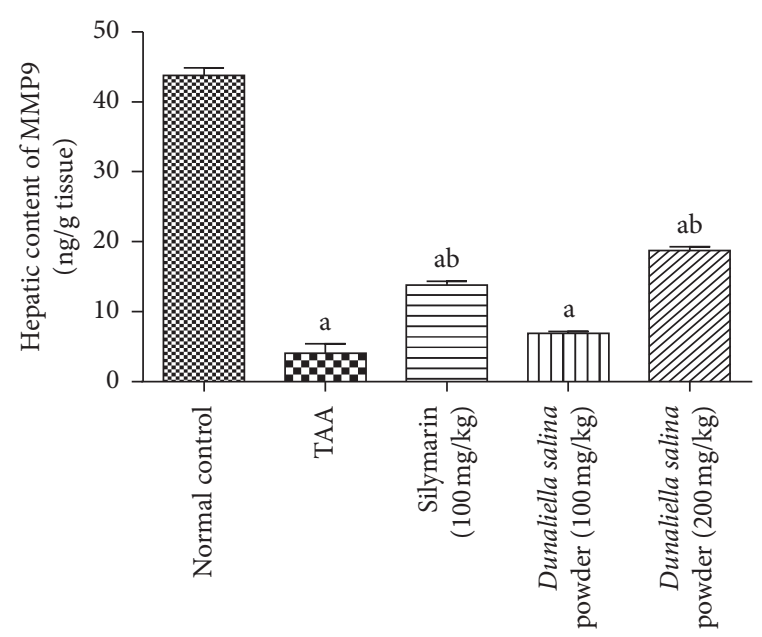

(c)

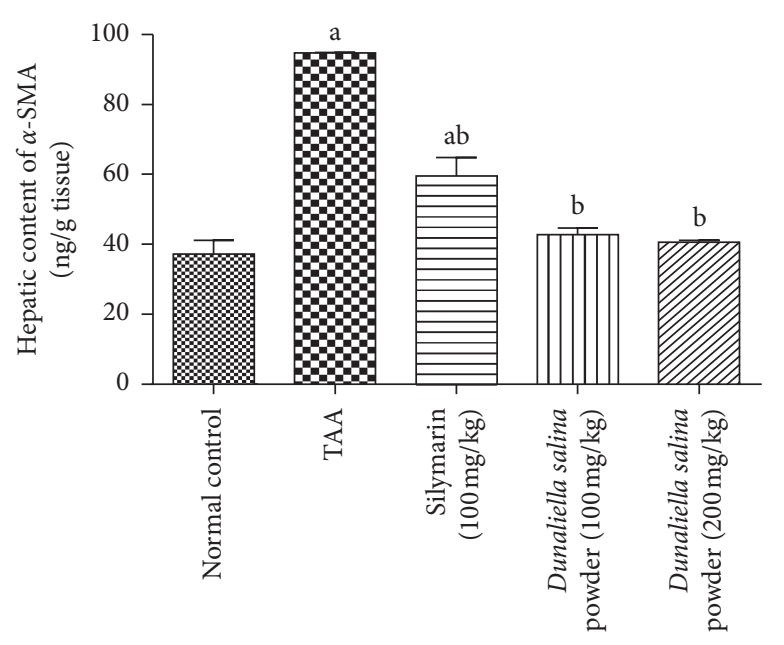

(b)

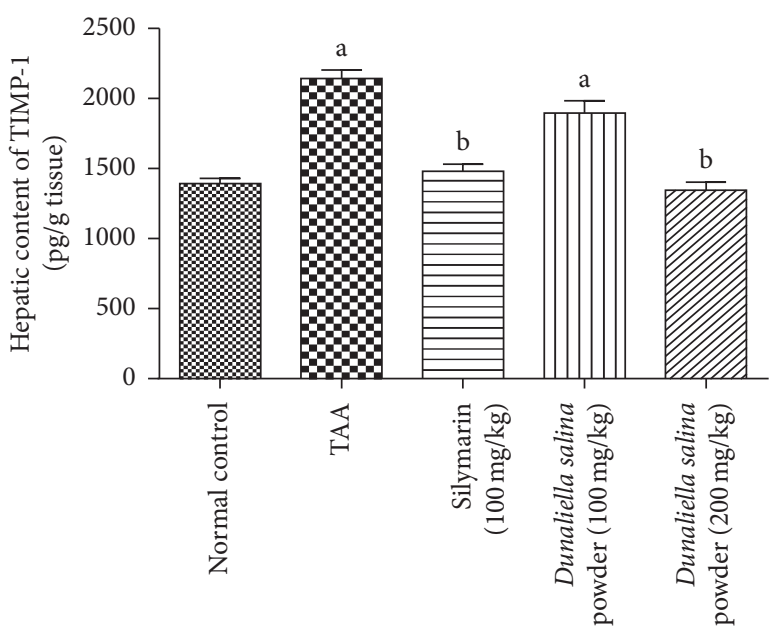

(d)

FIGURE 1: Effects of D. salina powder on hepatic contents of (a) collagen I, (b) SMA- $\alpha$, (c) MMP9, and (d) TIMP-1. Data are presented as mean \pm SE for each group $(n=8)$. Statistical analysis was carried out by one-way analysis of variance followed by Tukey's multiple comparisons test. 'Statistically significant from the control group and ${ }^{\mathrm{b}}$ statistically significant from the TAA group at $p<0.05$.

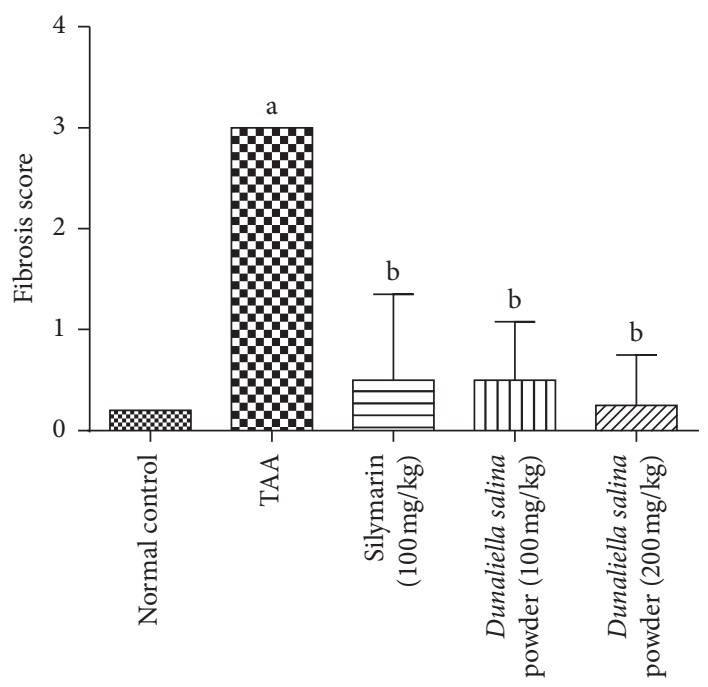

Figure 2: Effects of $D$. salina powder on fibrosis score. Data are presented as mean $\pm \mathrm{SE}(n=8)$. Statistical analysis was carried out by one-way analysis of variance followed by Tukey's multiple comparisons test. ${ }^{a}$ Statistically significant from the control group and ${ }^{\mathrm{b}}$ statistically significant from the TAA group at $p<0.05$. 


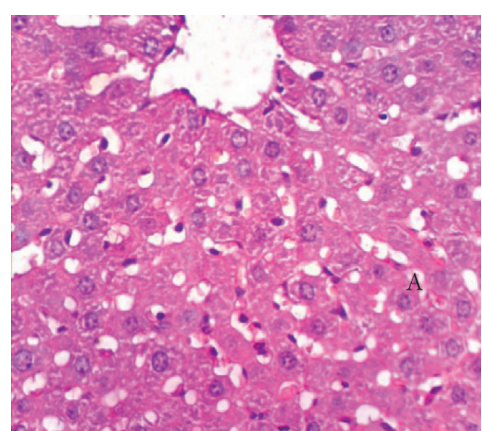

(a)

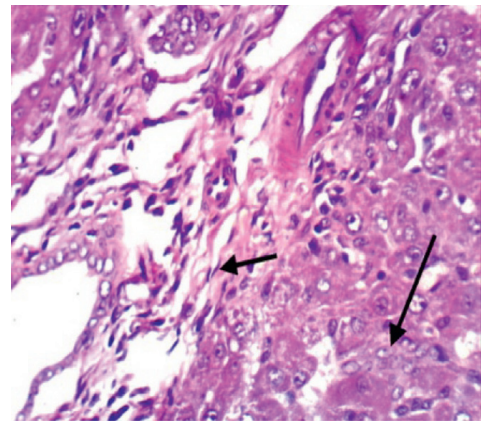

(c)

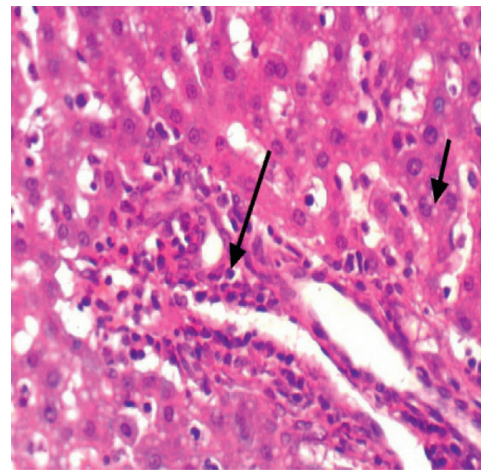

(e)

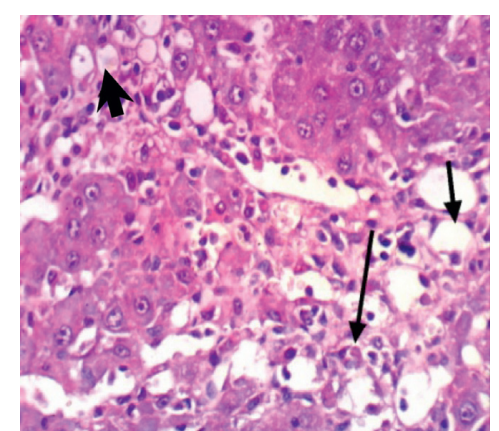

(b)

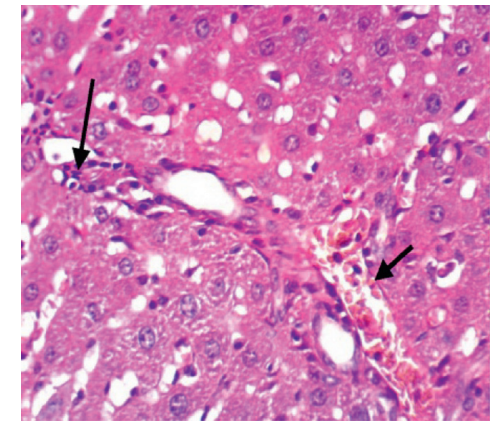

(d)

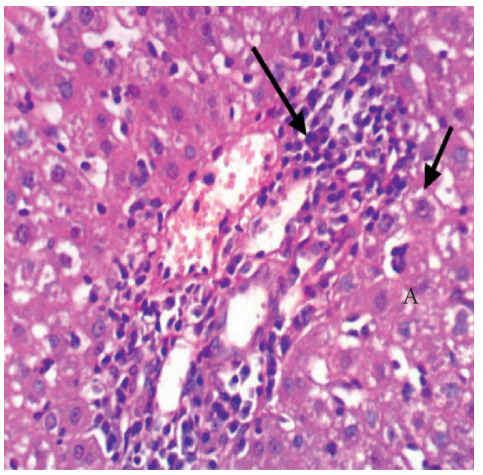

(f)

FIGURE 3: (a) Liver of control normal rat showing the normal histological structure of hepatic lobule (stage F0). (b) Liver of rat from the TAA group showing ballooning degeneration of hepatocytes (small arrow), fibrosis of hepatocytes (large arrow), and mononuclear cells infiltration (arrow head) (stage F3). (c) Liver of rat from TAA group showing portal fibrosis (small arrow) with oval cells proliferation (large arrow) (stage F3). (d) Liver of rat from silymarin-treated group showing fine strands of fibroblasts in portal tract (arrow) (stage F1). (e) Liver of rat from D. salina (100 mg/kg) showing fatty change of hepatocytes (small arrow) and portal infiltration with inflammatory cells (large arrow) (stage F1). (f) Liver of rat from D. salina $(200 \mathrm{mg} / \mathrm{kg})$ showing slight hydropic degeneration of hepatocytes (small arrow) and portal infiltration with inflammatory cells (large arrow) (stage F1) (H\&E, ×400).

TAA-induced liver fibrosis. Furthermore, the results of our study showed the decrease in MMP-9 expression was accompanied by increased TGF- $\beta 1$ expression in the TAA control group, and this result confirmed by previous hypothesis which stated that the downregulation of MMP-9 in the later stages of fibrogenesis appears to be mediated primarily by TGF- $\beta$, through a negative feedback mechanism [36] and by phosphoinositide 3-kinase (PI3K)-AKT signaling pathway [37].

Silymarin used to treat chronic liver disease in hepatofibrotic experimental animals and clinical studies [38]. However, silymarin absorption and its first-pass metabolism limited its treatment effect against severe liver damage [39], so alternative therapeutic agents from natural origin are needed as antihepatofibrotic agents. D. salina, a natural source of $\beta$-carotene, has a reversal role in TAAinduced liver fibrosis in rat. This study also highlights the regulation of two important pathways in fibrosis: inflammatory and fibrotic pathways. HSCs control the transport and storage of vitamin $\mathrm{A}$ and are named vitamin A-storing cells [40], and in the absence of these stores, a liver injury will provoke fibrosis and hepatic disease. It seems that reduced levels of $\beta$-carotene could stimulate hepatic cells damage [41]. D. salina contains 


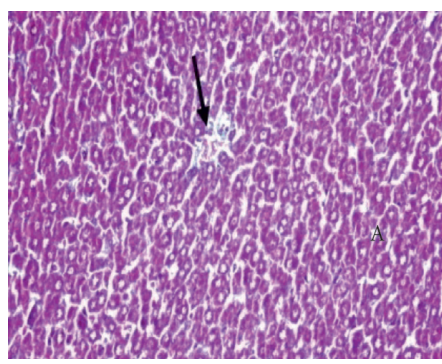

(a)

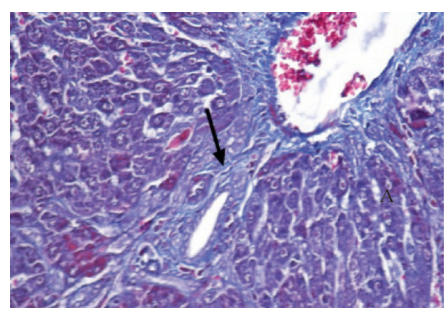

(c)

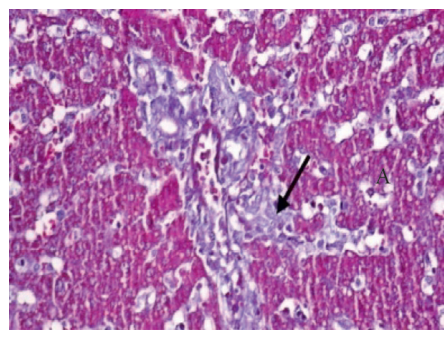

(e)

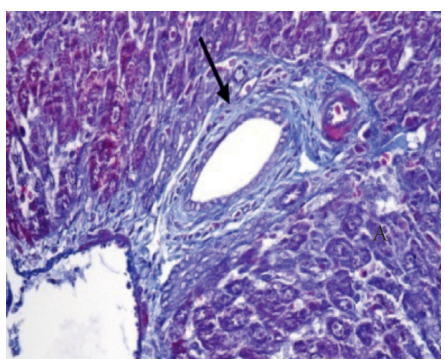

(b)

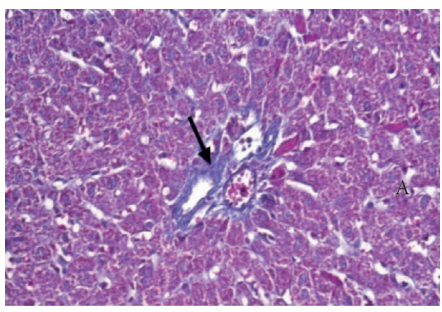

(d)

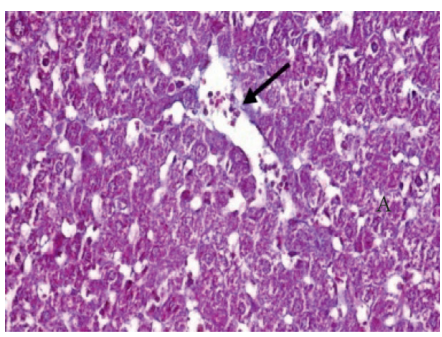

(f)

FIGURE 4: (a) Liver of control rat showing no histochemical reaction for collagen fibers deposition in the portal triad (F0). (b) Liver of rat from the TAA group showing strong positive histochemical reaction for collagen fibers deposition in the portal triad (F3). (c) Liver of rat from the TAA group showing strong positive histochemical reaction for collagen fibers deposition in the portal (F3). (d) Liver of rat treated with silymarin showing weak positive histochemical reaction for collagen fibers deposition (F1). (e) Liver of rat treated with $D$. salina $(100 \mathrm{mg} / \mathrm{kg})$ showing weak positive histochemical reaction for collagen fibers deposition (F1). (f) Liver of rat D. salina (200 mg/kg) showing no histochemical reaction for collagen fibers deposition (F0) (Masson's trichrome stain, $\times 400$ ).

$\beta$-carotene and may explain its benefits to treat fibrosis in this study.

\section{Conclusion}

The present study demonstrates that treatment with $D$. salina markedly ameliorates hepatic inflammation and fibrosis in rats. $D$. salina has a curative effective role in maintaining liver enzymes. Antifibrotic activity of $D$. salina is mediated through hepatic activation of MMP-9 with a suppression of TIMP-1 in the liver. Notably, D. salina exerts an anti-inflammatory activity by decreasing serum levels of TNF- $\alpha$, IL-6, and TGF- $\beta$.

\section{Data Availability}

The data used to support the findings of this study are available from the corresponding author upon request.

\section{Conflicts of Interest}

The authors declare that there are no conflicts of interest.

\section{Authors' Contributions}

Farouk El-Baz and Abeer Salama designed the study. Farouk El-Baz provided the materials. Abeer salama and Rania salama performed experimental part and biochemical analysis and drafted the manuscript. Abeer Salama analysed and interpreted the data and wrote the final draft of the article. Farouk El-Baz revised the final draft of the written article.

\section{Acknowledgments}

This work was supported by the alliance entitled "Integrated Pharmaceutical Alliance (IPA).” This alliance was funded by the Academy of Scientific Research and Technology under the "Egypt Research and Technology Alliances (EG-KTA) Program."

\section{References}

[1] V. Sanchez-Valle, N. C. Chavez-Tapia, M. Uribe, and N. Mendez-Sanchez, "Role of oxidative stress and molecular changes in liver fibrosis: a review," Current Medicinal Chemistry, vol. 19, no. 28, pp. 4850-4860, 2012. 
[2] S. L. Friedman, "Liver fibrosis-from bench to bedside," Journal of Hepatology, vol. 38, pp. S38-S53, 2003.

[3] R. Bataller and D. A. Brenner, "Liver fibrosis," Journal of Clinical Investigation, vol. 115, no. 2, pp. 209-218, 2005.

[4] S. L. Friedman, J. J. Maher, and D. M. Bissell, "Mechanisms and therapy of hepatic fibrosis: report of the AASLD single topic basic research conference," Hepatology, vol. 32, no. 6 , pp. 1403-1408, 2000.

[5] J. Kim, C.-W. Lee, E. K. Kim et al., "Inhibition effect of Gynura procumbens extract on UV-B-induced matrix-metalloproteinase expression in human dermal fibroblasts," Journal of Ethnopharmacology, vol. 137, no. 1, pp. 427-433, 2011.

[6] S. Duarte, J. Baber, T. Fujii, and A. J. Coito, "Matrix metalloproteinases in liver injury, repair and fibrosis," Matrix Biology, vol. 44-46, pp. 147-156, 2015.

[7] R. Visse and H. Nagase, "Matrix metalloproteinases and tissue inhibitors of metalloproteinases," Circulation Research, vol. 92, no. 8, pp. 827-839, 2003.

[8] R. R. Watson and V. Preedy, Bioactive Food as Dietary Interventions for Liver and Gastrointestinal Disease, Academic Press, Elsevier Inc., Atlanta, GA, USA, 1st edition, 2012.

[9] M. Ayyanar, K. Sankarasivaraman, and S. Ignacimuthu, "Traditional herbal medicines used for the treatment of diabetes among two major triabal groups in South Tamil Nadu, India," Ethnobotanical Leaflets, vol. 12, pp. 276-280, 2008.

[10] S. P. Tan, S. E. Parks, C. E. Stathopoulos, and P. D. Roach, "Extraction of flavonoids from bitter melon," Food and Nutrition Sciences, vol. 5, no. 5, pp. 458-465, 2014.

[11] M. Cohen-Naftaly and S. L. Friedman, "Current status of novel antifibrotic therapies in patients with chronic liver disease," Therapeutic Advances in Gastroenterology, vol. 4, no. 6, pp. 391-417, 2011.

[12] K. N. C. Murthy, A. Vanitha, J. Rajesha, M. M. Swamy, P. R. Sowmya, and G. A. Ravishankar, "In vivo antioxidant activity of carotenoids from Dunaliella salina-a green microalga," Life Sciences, vol. 76, no. 12, pp. 1381-1390, 2005.

[13] Y. Xu, I. M. Ibrahim, C. I. Wosu, A. Ben-Amotz, and P. J. Harvey, "Potential of new isolates of Dunaliella salina for natural beta-carotene production," Biology (Basel), vol. 7, no. 1, p. 14, 2018.

[14] F. K. El-Baz, R. A. Hussein, K. Mahmoud, and S. M. Abdo, "Cytotoxic activity of carotenoid rich fractions from Haematococcus pluvialis and Dunaliella salina microalgae and the identification of the phytoconstituents using LC-DAD/ESIMS," Phytotherapy Research, vol. 32, no. 2, pp. 298-304, 2018.

[15] F. K. El-Baz, R. A. Hussein, D. O. Saleh, and G. A. R. Abdel Jaleel, "Zeaxanthin isolated from Dunaliella salina microalgae ameliorates age associated cardiac dysfunction in rats through stimulation of retinoid receptors," Marine Drugs, vol. 17, no. 5, p. 290, 2019.

[16] C. F. Tsai, F. J. Lu, and Y. W. Hsu, "Protective effects of Dunaliella salina - a carotenoids-rich alga-against ultraviolet B-induced corneal oxidative damage in mice," Molecular Vision, vol. 18, pp. 1540-1547, 2012.

[17] T. Bai, Y. Yang, Y.-L. Wu et al., “Thymoquinone alleviates thioacetamide-induced hepatic fibrosis and inflammation by activating LKB1-AMPK signaling pathway in mice," International Immunopharmacology, vol. 19, no. 2, pp. 351-357, 2014.

[18] N. A. Afifi, A. Ramadan, W. El-Eraky, A. A. A. Salama, A. A. El-Fadaly, and A. Hassan, "Quercetin protects against thioacetamide induced hepatotoxicity in rats through decreased oxidative stress biomarkers, the inflammatory cytokines; (TNF- $\alpha),(\mathrm{NF}-\kappa \mathrm{B})$ and DNA fragmentation," Der Pharma Chemica, vol. 8, no. 9, pp. 48-55, 2016.

[19] H. M. Mansour, A. A. A. Salama, R. M. Abdel-Salam, N. A. Ahmed, N. N. Yassen, and H. F. Zaki, "The anti-inflammatory and anti-fibrotic effects of tadalafil in thioacetamide-induced liver fibrosis in rats," Canadian Journal of Physiology and Pharmacology, vol. 96, no. 12, pp. 1308-1317, 2018.

[20] M. T. Khayyal, F. K. El-Baz, M. R. Meselhy, G. H. Ali, and R. M. El-Hazeke, "Intestinal injury can be effectively prevented by Dunaliella salina in gamma irradiated rats," Heliyon, vol. 5, no. 5, Article ID e01814, 2019.

[21] F. K. El-Baz, H. F. Aly, and A. A. A. Salama, "Toxicity assessment of the green Dunaliella salina microalgae," Toxicology Reports, vol. 6, pp. 850-861, 2019.

[22] E. M. Brunt, "Grading and staging the histopathological lesions of chronic hepatitis: the Knodell histology activity index and beyond," Hepatology, vol. 31, no. 1, pp. 241-246, 2000.

[23] M. Kornek, E. Raskopf, I. Guetgemann et al., "Combination of systemic thioacetamide (TAA) injections and ethanol feeding accelerates hepatic fibrosis in $\mathrm{C} 3 \mathrm{H} / \mathrm{He}$ mice and is associated with intrahepatic up regulation of MMP-2, VEGF and ICAM-1," Journal of Hepatology, vol. 45, no. 3, pp. 370-376, 2006.

[24] Y.-S. Won, J.-W. Song, J.-H. Lim et al., "Genetically obese (ob/ ob) mice are resistant to the lethal effects of thioacetamide hepatotoxicity," Toxicology and Applied Pharmacology, vol. 291, pp. 38-45, 2016.

[25] S. A. Kumar, D. Tasdu, S. Gupta, and R. K. Bhardwaj Johri, "Reversal of hepatotoxin- induced prefibrogenic events by Emblica officinalis-a histological study," Indian Journal of Experimental Biology, vol. 45, no. 7, pp. 626-629, 2007.

[26] M. Galisteo, A. Suárez, M. P. Montilla, M. I. Fernandez, A. Gil, and M. C. Navarro, "Protective effects of Rosmarinus tomentosus ethanol extract on thioacetamide-induced liver cirrhosis in rats," Phytomedicine, vol. 13, no. 1-2, pp. 101-108, 2006.

[27] Y.-W. Hsu, C.-F. Tsai, W.-H. Chang, Y.-C. Ho, W.-K. Chen, and F.-J. Lu, "Protective effects of Dunaliella salina-a carotenoids-rich alga, against carbon tetrachloride-induced hepatotoxicity in mice," Food and Chemical Toxicology, vol. 46, no. 10, pp. 3311-3317, 2008.

[28] D. A. Renner, "Molecular pathogenesis of liver fibrosis," Transactions of the American Clinical and Climatological Association, vol. 120, pp. 361-368, 2009.

[29] A. Pellicoro, P. Ramachandran, J. P. Iredale, and J. A. Fallowfield, "Liver fibrosis and repair: immune regulation of wound healing in a solid organ," Nature Reviews Immunology, vol. 14, no. 3, pp. 181-194, 2014.

[30] T. Kisseleva, H. Uchinami, N. Feirt et al., "Bone marrowderived fibrocytes participate in pathogenesis of liver fibrosis," Journal of Hepatology, vol. 45, no. 3, pp. 429-438, 2006.

[31] A. M. Gressner, R. Weiskirchen, K. Breitkopf, and S. Dooley, "Roles of TGF-beta in hepatic fibrosis," Frontiers in Bioscience, vol. 7, no. 4, pp. 793-807, 2002.

[32] L. Zheng, L. Yin, L. Xu et al., "Protective effect of dioscin against thioacetamide-induced acute liver injury via FXR/ AMPK signaling pathway in vivo," Biomedicine \& Pharmacotherapy, vol. 97, pp. 481-488, 2018.

[33] D. Schmidt-Arras and S. Rose-John, "IL-6 pathway in the liver: from physiopathology to therapy," Journal of Hepatology, vol. 64, no. 6, pp. 1403-1415, 2016. 
[34] D. M. Bissell, S. L. Friedman, J. J. Maher, and F. J. Roll, "Connective tissue biology and hepatic fibrosis: report of a conference," Hepatology, vol. 11, no. 3, pp. 488-498, 1990.

[35] S. Robert, T. Gicquel, A. Bodin, V. Lagente, and E. Boichot, "Characterization of the MMP/TIMP imbalance and collagen production induced by IL-1beta or TNF-alpha release from human hepatic stellate cells," PLoS One, vol. 11, no. 4, Article ID e0153118, 2016.

[36] F. W.-T. Shek, R. C. Benyon, F. M. Walker et al., "Expression of transforming growth factor- $\beta 1$ by pancreatic stellate cells and its implications for matrix secretion and turnover in chronic pancreatitis," The American Journal of Pathology, vol. 160, no. 5, pp. 1787-1798, 2002.

[37] X.-J. Mi, J.-G. Hou, S. Jiang et al., "Maltol mitigates thioacetamide-induced liver fibrosis through TGF- $\beta 1$-mediated activation of PI3K/akt signaling pathway," Journal of Agricultural and Food Chemistry, vol. 67, no. 5, pp. 1392-1401, 2019.

[38] N. Vargas-Mendoza, E. Madrigal-Santillan, A. MoralesGonzalez et al., "Hepatoprotective effect of silymarin," World Journal of Hepatology, vol. 6, no. 3, pp. 144-149, 2014.

[39] A. M. Mohsen, M. H. Asfour, and A. A. A. Salama, "Improved hepatoprotective activity of silymarin via encapsulation in the novel vesicular nanosystem bilosomes," Drug Development and Industrial Pharmacy, vol. 43, no. 12, pp. 2043-2054, 2017.

[40] H. Senoo, K. Imai, Y. Mezaki et al., "Accumulation of vitamin A in the hepatic stellate cell of arctic top predators," The Anatomical Record: Advances in Integrative Anatomy and Evolutionary Biology, vol. 295, no. 10, pp. 1660-1668, 2012.

[41] M. Kankofer and E. Albera, "Postpartum relationship of beta carotene and vitamin A between placenta, blood and colostrum in cows and their newborns," Experimental and Clinical Endocrinology \& Diabetes, vol. 116, no. 7, pp. 409-412, 2008. 